\title{
Basic and Clinical Cancer Research in the Healthcare Reform Era
}

\section{Jimmy Thomas Efird*}

Departments of Public Health and Cardiovascular Sciences (East Carolina Heart Institute), Brody School of Medicine, Center for Health Disparities Research, East Carolina University, 405 Rotary Avenue, Greenville, North Carolina 27858, USA

On March 23, 2010, President Obama signed the Affordable Care Act (ACA) into law [1]. The overall vision of this important legislation is to ensure that all individuals in the United States (US), regardless of income, race, and gender, have equal opportunity to live long, healthful, and productive lives. The bill strengthens Medicare by adding new benefits, combatting healthcare fraud, reducing costs, and improving care for patients. A key requirement of the bill is that at least $80 \%$ of premium dollars paid to insurers be spent on medical care and quality improvements. Over the next 10 years, implementation of the ACA will reduce the US budget by $\$ 210$ billion and by more than $\$ 1$ trillion over the following decade. A family of four in 2014 is expected to save up to $\$ 2,300$ on their premium attributable to the ACA.

Translating science to better care in a timely and efficient manner will be critical in delivering affordable care to the American public. The challenge is multifaceted and complex in nature. There will be many obstacles to overcome if the ACA is to be genuinely inclusive of the underserved and increasingly diverse population in the US. In the field of cancer research and therapy, this will entail advancing the training and funding opportunity of scientists and clinicians to deliver the technologic and system breakthroughs of tomorrow. Broadly, this will involve developing new and more effective strategies for transforming translational and clinical research into cost-effective patient care, based on rigorous scientific evidence and replication across population strata.

Cancer is the second most common cause of death in the US with an estimated 1,600 people succumbing to this disease each day [2]. The annual price tag for cancer exceeds $\$ 226$ billion, representing roughly $1.5 \%$ of the US Gross Domestic Product (GDP) [3]. Direct medical costs for treatment alone account for almost half of the national bill ( $\$ 103$ billion), while costs of lost productivity make up the remaining amount [3]. On a global scale, the indirect economic burden of cancer ( $\$ 895$ billion) is nearly 20\% higher than heart disease ( $\$ 753$ billion), with 83 million years of "healthy life" lost due to death and disability from cancer [4].

Over the last 20 years, total medical costs of cancer in the US have doubled, shifting from the inpatient to the outpatient setting [5]. The bulk of the increase is attributable to the number of new cancers diagnosed among the aging population base and increasing cancer prevalence [5]. A disproportionate amount of healthcare costs occur in the months before death [5]. Accordingly, the absolute number of individuals affected and the overall cost of cancer is expected to significantly increase as the US population ages and the prevalence of cancer becomes increasing skewed toward the later decades of life. The gravity of the situation likely will expand because of improvements in cardiovascular treatment and subsequent reductions in mortality due to this and other competing risks. Reversing this trend will depend on improving the effectiveness of early cancer detection and treatment. Advances in cancer biomarkers and targeted therapies also will help reduce costs by containing cancer at an early stage of development. Other strategies will involve implementing life-course public health interventions and developing more cost-efficient models for outpatient treatment of cancer such as increasing the number of community- based clinics and the broader use of nurse practitioners. The average costs for cancer drugs often exceed the costs of drugs to treat other illnesses and this represents another venue for lowering costs through better drug development and increased use of bioequivalent generics. The impact of these and other changes aimed at reducing cancer costs will need to be carefully monitored and fine-tuned as additional data becomes available.

Cancer represents a disease of many intertwined etiologies and multiple causal pathways. Understanding cancer and containing costs associated with this tragic disease will necessitate collaboration among bench and clinical scientists, as well as epidemiologists and socialbehavioral researchers. Achieving the goal of reducing the incidence/ prevalence and price tag of cancer remains a daunting challenge. But solutions are obtainable if we critically evaluate present day paradigms and set about to constructively alter the landscape of future prevention, diagnosis and treatment of cancer. Recognizing exciting new trends and proactively realigning resources will be necessary if we are to leverage the benefit of promising scientific advances and make substantial inroads in our battle against cancer.

Bridges to the future will need to be built that promotes strong partnerships among the sciences and lay the foundation for innovative funding and reimbursement models, if we are to accomplish our mission. Learning from experts within and outside the field, being persistent, and thinking "outside the box" will lead to unforeseen and monumental solutions as we move into the era of healthcare reform. Emphasis will need to be placed on integrative and transformative approaches, consensus building, and putting forth leadership models that are flexible enough to accommodate future changes in the system. This will include offering guiding principles as well as concrete objectives and action steps.

Much of the improvements also will need to come from beyond the traditional laboratory setting to include changes in lifestyle and living conditions aimed at decreasing the onslaught and severity of cancer. It will be important to focus on not only individual determinants of cancer such as stress, poor diet, lack of exercise and inadequate self-care, and smoking, but also on social-cultural/environmental determinants including health disparities, poverty, adverse living

*Corresponding author: Jimmy Thomas Efird, Ph.D., M.Sc., Departments of Public Health and Cardiovascular Sciences (East Carolina Heart Institute) Brody School of Medicine, Center for Health Disparities Research, East Carolina University, 405 Rotary Avenue, Greenville, North Carolina 27858, USA, Tel: 650.248.8282; E-mail: Jimmy.efird@stanfordalumni.org

Received December 17, 2012; Accepted December 19, 2012; Published December 21, 2012

Citation: Efird JT (2013) Basic and Clinical Cancer Research in the Healthcare Reform Era. J Cancer Sci Ther 5: e119. doi:10.4172/1948-5956.1000e119

Copyright: (c) 2013 Efird JT. This is an open-access article distributed under the terms of the Creative Commons Attribution License, which permits unrestricted use, distribution, and reproduction in any medium, provided the original author and source are credited. 
Citation: Efird JT (2013) Basic and Clinical Cancer Research in the Healthcare Reform Era. J Cancer Sci Ther 5: e119. doi:10.4172/19485956.1000 e119

Page 2 of 2

condition, and cultural insensitivities. Racial and ethnic minorities and those who live in increasingly economically disparate settings continue to suffer a disproportionate share of the cancer burden and death in the US. They are more likely to be diagnosed with cancer at an advanced stage, requiring more extensive and costly therapy [3]. Inequalities in the societal response to cancer health are not predestined but rather are malleable and subject to change if the will and determination exists to do so. Almost 51 million Americans were uninsured in 2009 (one-third of Hispanics and one in 10 children 17 years of age and younger), a number that is expected to decrease significantly as the ACA is rolled out [3]. While healthcare quality and access are suboptimal for many Americans today, especially those from minority and low income groups, the ACA gives hope for the future of cancer prevention and care.

\section{References}

1. The White House (2012) A more secure future: what the new health law means for you and your family.

2. American Cancer Society (2012) Economic Impact of Cancer.

3. American Cancer Society (2012) Cancer Facts \& Figures 2012. Atlanta.

4. American Cancer Society and Livestrong (2012) The Global Economic Cost of Cancer.

5. Tangka F, Trogdon J, Richardson L, Howard D, Sabatino S, et al. (2010) Cancer treatment cost in the United States. Cancer 116: 3477-3484. 